\title{
Collective Radiative Dynamics of an Ensemble of Cold Atoms Coupled to an Optical Waveguide
}

\author{
Riccardo Pennetta $\odot,{ }^{*}$ Martin Blaha $\odot$, Aisling Johnson $\odot,{ }^{\dagger}$ Daniel Lechner®, \\ Philipp Schneeweiss $\odot$, Jürgen Volz๑, and Arno Rauschenbeutel $\odot^{\ddagger}$ \\ Department of Physics, Humboldt-Universität zu Berlin, 12489 Berlin, Germany
}

(Received 7 September 2021; accepted 29 November 2021; published 16 February 2022)

\begin{abstract}
We experimentally and theoretically investigate collective radiative effects in an ensemble of cold atoms coupled to a single-mode optical nanofiber. Our analysis unveils the microscopic dynamics of the system, showing that collective interactions between the atoms and a single guided photon gradually build up along the atomic array in the direction of propagation of light. These results are supported by time-resolved measurements of the light transmitted and reflected by the ensemble after excitation via nanofiber-guided laser pulses, whose rise and fall times are shorter than the atomic lifetime. Superradiant decays more than 1 order of magnitude faster than the single-atom free-space decay rate are observed for emission in the forward-propagating guided mode, while at the same time, no speed-up of the decay rate is measured in the backward direction. In addition, position-resolved measurements of the light that is transmitted past the atoms are performed by inserting the nanofiber-coupled atomic array in a 45-m-long fiber ring resonator, which allow us to experimentally reveal the progressive growth of the collective response of the atomic ensemble. Our results highlight the unique opportunities offered by nanophotonic cold atom systems for the experimental investigation of collective light-matter interaction.
\end{abstract}

DOI: 10.1103/PhysRevLett.128.073601

The study of collective effects among quantum emitters has its roots in the seminal work of Dicke [1], which provides a clear formalism to calculate the spontaneous emission of a cloud of $N$ two-level atoms confined in a volume with dimensions small compared to the emitted wavelength $\lambda$. In the case of a single excitation in the system, superradiant emission can be observed for an ensemble prepared in the so-called Dicke state: $|D\rangle=(1 / \sqrt{N}) \sum_{n=1}^{N}|n\rangle$, where the notation $|n\rangle$ indicates that the $n$th atom is excited and the others are in the ground state [see Fig. 1(a)]. While in the Dicke state the excitation is equally shared in the ensemble, in general terms, superradiance does not require indistinguishability of the atoms, but rather of the emitted photons. In other terms, all atoms must couple to the same optical mode.

From this perspective, it is not surprising that many of the phenomena first described in Ref. [1] persist even in the case of extended ensembles [2], a situation closer to many experimental configurations. For instance, superand subradiance have been reported in cold atom clouds [3-10], Rydberg atoms [11,12], and ensembles of nuclei [13]. Under these circumstances, excitation through the

Published by the American Physical Society under the terms of the Creative Commons Attribution 4.0 International license. Further distribution of this work must maintain attribution to the author(s) and the published article's title, journal citation, and DOI. absorption of a photon with wave vector $\mathbf{k}$ is more appropriately described by a so-called timed Dicke state [2]: $|\mathrm{TD}\rangle=(1 / \sqrt{N}) \sum_{n=1}^{N} e^{i \mathbf{k} \cdot \mathbf{r}_{n}}|n\rangle$, in which $\mathbf{r}_{n}$ indicates the position of the $n$th atom. Compared to the ordinary Dicke state, the introduction of the spatial phase factors breaks the symmetry of the state, causing a different dynamics for each of the atoms in the ensemble. Moreover, the system experiences an enhanced collective emission of light with wave vector $\mathbf{k}$ (i.e., in the same optical mode that excited the system) for which the different emission amplitudes interfere constructively [2] [see Fig. 1(b)]. Recent theoretical studies successfully

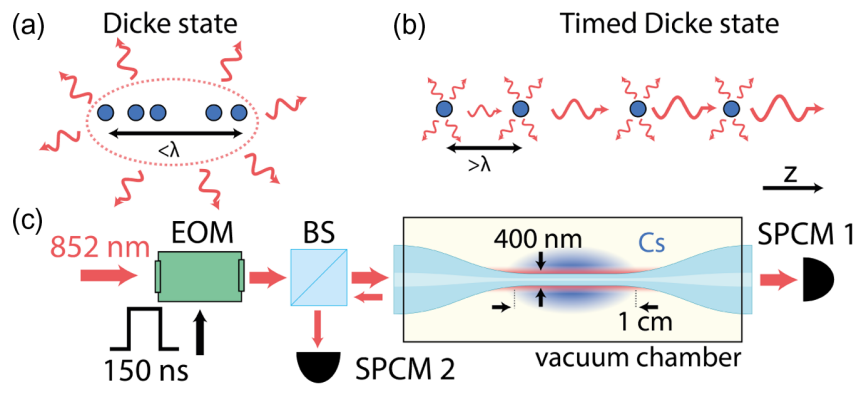

FIG. 1. (a),(b) Sketch of a one-dimensional atomic ensemble prepared in (a) the Dicke state and (b) the timed Dicke state and corresponding emission properties. (c) Experimental setup: EOM, electro-optic amplitude modulator; BS, beam splitter; SPCM, single-photon counting module; Cs, cesium. 
describe the nontrivial time evolution of this state for a three-dimensional disordered atom cloud $[14,15]$; however, the complexity of this configuration often hinders an intuitive understanding of the dynamics of its microscopic constituents.

In this Letter, we study experimentally and theoretically collective radiative effects in an ensemble of cold atoms coupled to a single-mode optical waveguide. A theoretical analysis based on a real-space quantum mechanical approach [16] allows a clear microscopic (i.e., atom per atom) description of phenomena such as superradiance and collective multimode Rabi oscillations [17]. In particular, we show that the cascaded interaction among the atoms and a single guided photon causes a gradual buildup of the collective effects along the atomic ensemble in the direction of propagation of light. In contrast with the traditional Dicke description, this dynamics is independent of the interatomic distance (except for atoms arranged at the Bragg condition).

We experimentally support these predictions by interfacing a cloud of laser-cooled cesium (Cs) atoms with guided photons in the evanescent field of an optical nanofiber. This configuration allows us to couple thousands of atoms, whose average separation is greater than $\lambda$, to a single and well-defined guided mode and therefore represents an ideal candidate to investigate the physics of the timed Dicke state. We explore the temporal response of the system by exciting the atoms with boxcar-shaped pulses of nanofiber-guided light, whose rise and fall times are much shorter than the atomic lifetime, and recording the power of the light that is transmitted and reflected by the ensemble. We experimentally reveal the progressive growth of collective effects by measuring the temporal dynamics of a single optical pulse propagating multiple times through the ensemble. This measurement, which we demonstrate to be equivalent to a single passage through atomic subsets tens of meters away from each other, allows us to capture experimentally the position-dependent increase of the superradiant decay rate predicted by our model.

The theoretical framework used to describe light-matter interaction in our system is detailed in the Supplemental Material [18]. Briefly, following the approach of Refs. $[16,19]$, we start with calculating the transmission amplitude in the steady state for $N$ atoms for a single frequency excitation:

$$
t_{N}(\Delta)=\prod_{n=1}^{N} t_{\mathrm{at}, n}(\Delta)=\prod_{n=1}^{N}\left[1-\frac{\beta_{n} \Gamma_{0}}{\frac{\Gamma_{0}}{2}+i \Delta}\right],
$$

where $\Delta=\omega-\omega_{a}$ is the laser-atom detuning, $t_{\mathrm{at}, n}$ is the transmission amplitude of the $n$th atom, and $\beta_{n}$ indicates the ratio of the intrinsic spontaneous emission rate of the $n$th atom into the waveguide and the single-atom total emission rate $\Gamma_{0}$. The excited state amplitude of the $n$th atoms is proportional to the local light field and can be calculated as

$$
\phi_{n}(\Delta)=i \frac{\sqrt{v_{g}}}{\sqrt{\beta_{n} \Gamma_{0}}}\left[t_{n}(\Delta)-t_{n-1}(\Delta)\right] u_{\mathrm{in}}(\Delta) e^{i k z_{n}},
$$

where $v_{g}$ is the group velocity of the waveguide mode, $u_{\text {in }}(\Delta)$ is the scalar field amplitude of the excitation pulse, and $z_{n}$ indicates the position of the $n$th atom. Equation (2) illustrates that the phase of the excited state amplitude differs for each atom and is given by $\varphi=\arg \left[t_{n}(\Delta)-\right.$ $\left.t_{n-1}(\Delta)\right]+k z_{n}$. The first term results from the dispersive property of the atoms and vanishes for far-detuned light $\left(\Delta \gg \Gamma_{0}\right)$ and on resonance. In this case, $\varphi=k z_{n}$, i.e., the phase of the timed Dicke state.

In the linear regime, the time dynamics of the transmitted optical field $u_{t}(t)$ can be calculated as

$$
u_{t}(t)=\mathcal{F}^{-1}\left[u_{\text {in }}(\Delta) t_{N}(\Delta)\right],
$$

where $\mathcal{F}^{-1}$ indicates the inverse Fourier transform. A similar analysis also allows us to estimate the time evolution of the excited state amplitudes and the reflected light (see Supplemental Material [18]). Note that Eqs. (1) and (3) are independent of the position of the single atoms.

The experimental setup is sketched in Fig. 1(c). A cold cloud of $\mathrm{Cs}$ atoms from a magneto-optical trap is prepared around a single-mode optical nanofiber (diameter $\approx 400 \mathrm{~nm}$, waist length $\approx 1 \mathrm{~cm}$ ). The atoms are probed on the Cs $D 2$ transition $\left(6 S_{1 / 2}, F=4 \rightarrow 6 P_{3 / 2}, F^{\prime}=5\right)$ with 150 -ns-long pulses of nanofiber-guided light. The pulses are generated with an electro-optic amplitude modulator (EOM) based on a Mach-Zehnder interferometer and have rise and fall times $(\approx 850 \mathrm{ps})$ that are short compared to the lifetime of the excited state $\left(2 \pi / \Gamma_{0}=30.4 \mathrm{~ns}\right.$, $\Gamma_{0} / 2 \pi=5.2 \mathrm{MHz}$ [20]). The average value of $\beta=$ $0.55 \%$ [21]. The power of the light transmitted and reflected by the atomic ensemble is recorded using two single-photon counting modules (SPCMs). Each excitation pulse has a mean power much smaller than one singlephoton energy per atomic lifetime, placing our experiment in the linear optics (i.e., low saturation) regime.

Figure 2 shows typical time-resolved measurements of the transmitted power for an optical depth (OD) of 19.3 (corresponding to $\approx 900$ atoms) and laser detuning $\Delta$ from the atomic transition of $17.3 \Gamma_{0}$ [Fig. 2(a)] and $5.7 \Gamma_{0}$ [Fig. 2(b)]. At the leading edge of the transmitted pulse, pronounced Rabi oscillations appear, which, in contrast to the single-atom case, are not simple damped sinusoidal functions, as can be seen, e.g., from the subsequent revivals marked in Fig. 2(a). These features are a clear signature of collective interaction among the atoms [17]. Theoretical predictions (without free parameters) are depicted as solid red lines, and their agreement with the measurements is excellent.

To clarify the microscopic dynamics of the system, Figs. 2(c) and 2(d) show the calculated time evolution of the excitation probability of the 1st, 100th, and 600th atom 

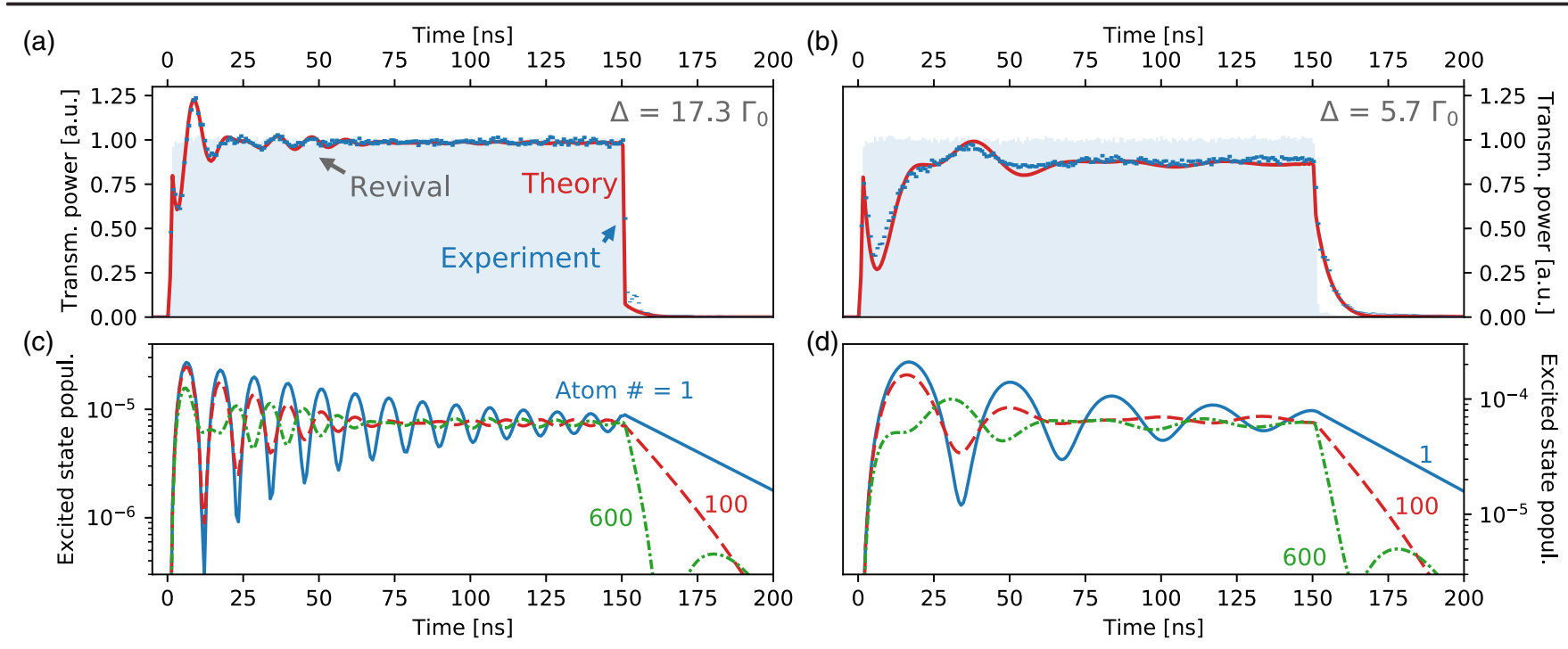

FIG. 2. (a),(b) Measured transmission for $\mathrm{OD}=19.3$ at a detuning of (a) $17.3 \Gamma_{0}$ and (b) $5.7 \Gamma_{0}$. The blue dots represent the measured data, while the theoretically predicted transmission is shown as a solid red line. The blue shaded area illustrates the measured pulse shape in the absence of atoms. (c),(d) Calculated probability of finding the first (solid blue line), 100th (dashed red line), and 600th atom (dashdotted green line) of the ensemble in the excited state as a function of time for the same experimental parameters of (a) and (b).

in the array (a more detailed plot can be found in the Supplemental Material [18]). Two features are clearly noticeable: First, while the ensemble is illuminated by the probing pulse, each atom undergoes Rabi oscillations with similar frequency (approximately equal to $\Delta$ ) but very different amplitude, phase, and damping rate. At the beginning of the pulse, all atoms start to oscillate in phase with each other; however, the Rabi oscillations remain sinusoidal only for the first atom in the array, which behaves as if it was completely isolated from the others. The successive atoms driven by the field that results from the interference between the probe pulse and the light emitted by all the previous atoms eventually reverse their oscillation phase. This process may repeat itself several times for the atoms toward the end of the array. This complex dynamics is at the origin of the peculiar temporal response measured in the experiment. Second, following the switch off of the excitation pulse, even if the ensemble is approximately uniformly excited, the decay rate differs from atom to atom, being equal to the intrinsic rate $\Gamma_{0}$ for the first atom and becoming increasingly superradiant (i.e., $\Gamma>\Gamma_{0}$ ) for the subsequent ones.

To experimentally investigate the superradiant behavior in more detail, we measure the transmitted light after the switch off of the excitation pulse as a function of the OD and infer its initial decay rate from an exponential fit (see Supplemental Material [18]). In the following, we refer to this quantity as the pulse decay rate. Figure 3 illustrates our results obtained with a laser detuning of $\Delta=3.8 \Gamma_{0}$ and compares them with our theoretical predictions. The latter have been obtained by carrying out the same fitting procedure on the calculated transmitted pulse. We observe a speed-up of the pulse decay rate of about 1 order of magnitude and an approximately linear dependence on the OD. We would like to underline that the speed-up of the pulse decay rate is not a direct indicator of superradiance. The collective decay rate of the ensemble is defined as $\Gamma_{\text {coll }}(t)=-\dot{E}(t) / E(t)$, where $E$ is the total energy stored in the atoms. Therefore, to calculate $\Gamma_{\text {coll }}$ we have to average the individual decay rates of the single atoms weighted by their excited state populations [see Figs. 2(c) and 2(d)]. The red line in Fig. 3 shows the calculated $\Gamma_{\text {coll }}$ at the switch off of the excitation pulse as a function of the OD. One can see that, for the parameters used in our experiment, only for small OD is the pulse decay rate a good approximation of $\Gamma_{\text {coll }}$.

The difference between the pulse and ensemble decay rate becomes even more evident if we compare them as a function of the laser detuning, as shown in Fig. 4 for

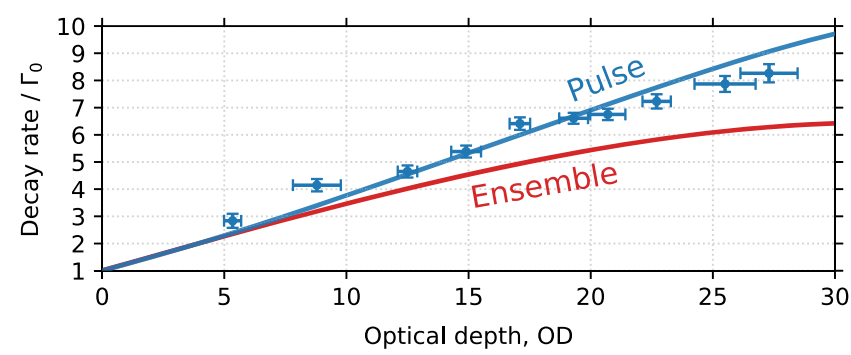

FIG. 3. Measured (blue dots) and calculated (solid blue line) pulse decay rate, i.e., the decay rate of the transmitted optical power right after the switch off of the excitation pulse, as a function of the OD for a detuning of $\Delta=3.8 \Gamma_{0}$. The solid red line indicates the collectively enhanced ensemble decay rate $\Gamma_{\text {coll }}$, i.e., the excited state population decay rate averaged over the ensemble. 


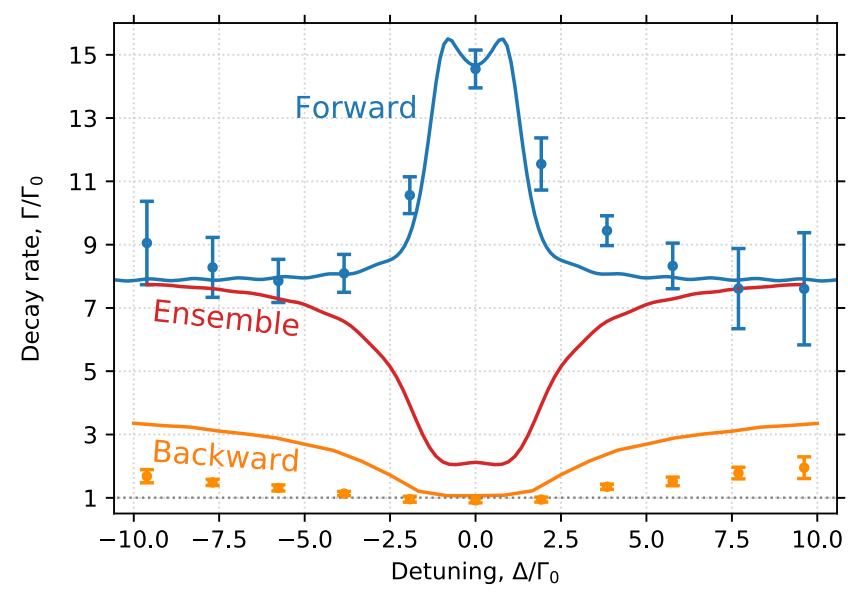

FIG. 4. Measured and predicted pulse decay rate in the forward (blue dots and line) and backward direction (orange dots and line) as a function of the laser detuning for $\mathrm{OD}=26$. The solid red line depicts the calculated collectively enhanced ensemble decay rate $\Gamma_{\text {coll }}$.

$\mathrm{OD}=26$. Counterintuitively, on resonance the difference between these two quantities is the largest. In this case, the ensemble is opaque, and all atoms toward the end of the array are initially not excited and, therefore, do not contribute to the collective enhancement of the ensemble decay rate. However, because of their strong reabsorption, they cause a fast decrease of the light power leaving the ensemble, which results in a fast pulse decay rate. Increasing the laser detuning allows one to excite a larger fraction of all atoms, leading to significantly larger ensemble decay rates.

To further test our physical picture, in Fig. 4 we also compare the pulse decay rates of the light scattered in the forward and backward direction. While we measure superradiant decay rates in the forward direction close to resonance, the back-reflected light decays with the intrinsic rate $\Gamma_{0}$. This asymmetry arises because the detected backward-propagating photons are mostly emitted by atoms at the beginning of the array. Indeed, in a first approximation, the probability that a photon emitted from the $n$th atom is detected in the backward direction is $\approx r t^{2(n-1)}$, which decays exponentially with $n$. Here, $r$ and $t$ are the singleatom reflection and transmission coefficients (see Supplemental Material [18]). Detuned excitation pulses experience a weaker light-atom coupling, allowing photons reflected by atoms located deeper in the ensemble to reach the detector and leading to an increase in the measured decay rate of the back-reflected pulse. This behavior is qualitatively reproduced by our model (solid line in Fig. 4). We attribute the discrepancies between the predicted and observed decay rate to inhomogeneous broadening of the atomic transition frequencies and excited state decay rates, e.g., due to the close vicinity of the atoms to the nanofiber surface [22]. Its effects are negligible for the forward pulse propagation, which is dominated by the collective response of the atomic ensemble.

As mentioned, our analysis shows that collective effects in the ensemble build up gradually along the propagation direction of light and are independent of the interatomic distance. This holds true even when the separation among the atoms significantly exceeds the spatial extent of the excitation pulse and the distance traveled by light in an atomic lifetime. Our nanofiber-based atom-light interface is a perfect candidate to access this regime, which, experimentally unexplored, has been subject of recent theoretical investigations $[15,23]$.

With this in mind, we place the nanofiber in an $\approx 45$-mlong fiber ring resonator [21,24], whose cavity round-trip time (220 ns) is much longer than the excitation pulse duration (reduced to $120 \mathrm{~ns}$ in the following). Under these circumstances, the ring resonator does not provide any field enhancement but rather enables multiple passes of the probe pulse through the ensemble. At each subsequent round-trip, part of the light is out coupled using a fiber coupler and detected with a SPCM [see Fig. 5(a)]. This experimental configuration allows us to perform positionresolved measurements of the growth of collective effects in an ensemble consisting of several atomic ensembles $45 \mathrm{~m}$ away from each other. It is interesting to note that, since the average time of flight of the atoms through the evanescent field of the nanofiber $(\approx 1 \mu \mathrm{s}[25])$ exceeds the cavity round-trip time, the collective effects in this experiment originate from the interaction among an atomic ensemble and its time-delayed counterparts. Nonetheless, since the interval between adjacent pulses is long enough for the atom cloud to completely decay into its ground state, our results after the $m$ th round-trip are equivalent to what could be observed with a single propagation (i.e., no optical cavity) through an ensemble with optical depth $\mathrm{OD}_{\text {tot }}=m \times \mathrm{OD}_{\mathrm{SP}}$, where $\mathrm{OD}_{\mathrm{SP}}$ is the single-pass $\mathrm{OD}$.

Figures 5(b)-5(h) depict the measured out-coupled power for the first seven cavity round-trips for $\mathrm{OD}_{\mathrm{SP}}=$ 14 and a detuning of $\Delta=8.7 \Gamma_{0}$, which was obtained by averaging over $9 \times 10^{6}$ excitation pulses. The pulse switchon dynamics is characterized by a growing complexity of the Rabi oscillations, which, round-trip after round-trip, increasingly deviate from the sinusoidal single-atom behavior. In particular, for very large OD [see Figs. 5(e)-5(h)], new oscillations appear, whose frequency is significantly faster than $\Delta$ and strongly depends on the OD, a regime qualitatively different from the one shown in Fig. 2 and discussed in Ref. [17]. At a microscopic level, this is due to the large light-matter coupling strength which causes the atoms toward the end of the array to reverse their oscillation phase before a single Rabi cycle is completed; see Supplemental Material [18].

The trailing edge of the pulses exhibits superradiant decay rates, as shown in Fig. 5(i). Beyond $\mathrm{OD}_{\text {tot }}=56$, we observe a change in the pulse shape and the appearance of a 
(a)

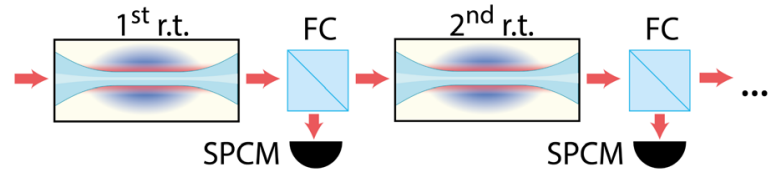

(b)

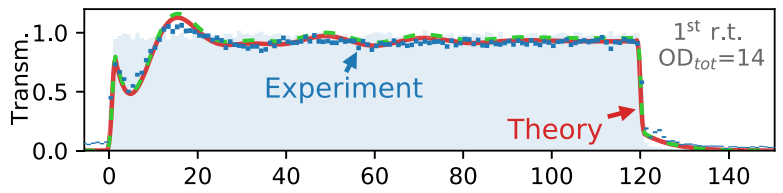

(c)



(d)



(e)

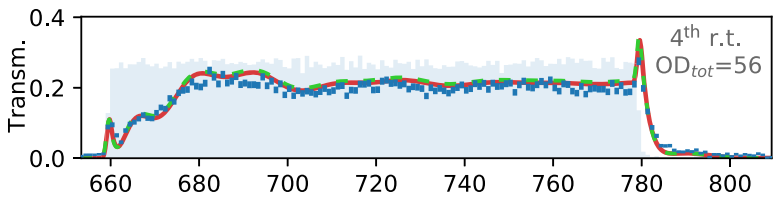

(f)



(g)



(h)

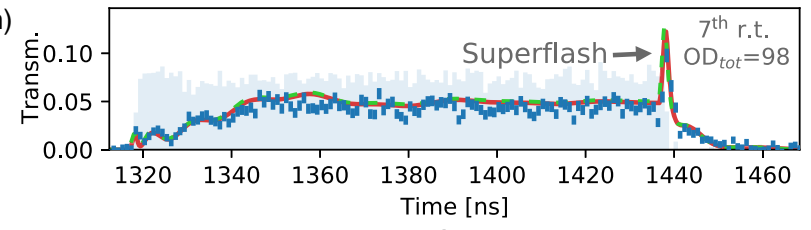

(i)

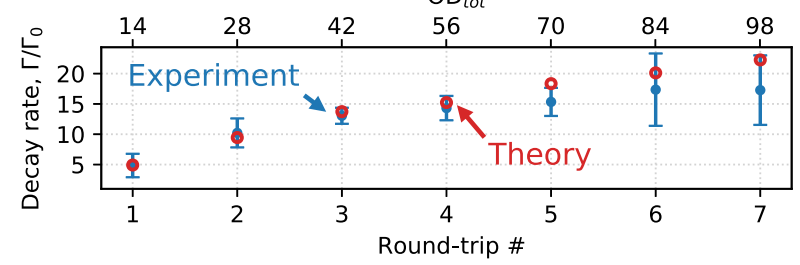

FIG. 5. (a) Simplified schematic of the propagation of the optical pulse when the nanofiber is inserted in an $\approx 45$-m-long fiber ring resonator (FC, fiber coupler; SPCM, single-photon counting module; r.t., round-trip). (b)-(h) Measured transmitted optical power of 120-ns-long probe pulses for the first seven cavity round-trips $\left(\mathrm{OD}_{\mathrm{SP}}=14\right.$ and detuning of $\left.\Delta=8.7 \Gamma_{0}\right)$. The solid red lines depict the theoretical prediction when the presence of the optical cavity is included in the system Hamiltonian. For comparison, the green dashed lines are single-pass calculations for propagation through an optical depth $\mathrm{OD}_{\text {tot }}=m \times \mathrm{OD}_{\mathrm{SP}}$, where $m$ indicates the round-trip number. (i) Measured (blue dots) and calculated (red circles) pulse decay rate as a function of the cavity round-trip. shoulder in the collectively emitted light field [Figs. 5(e)5(h)]. The latter can be understood considering that for very large atom number, the slower decay of the atoms early in the array can reexcite the successive atoms, which then decay again at a later time. In addition, the experiment reveals the progressive appearance of a coherent superflash of light (as referred to in Ref. [26]), whose peak intensity is larger than the one of the exciting pulse [see Figs. 5(e)-5(h)].

The theoretical predictions shown in Figs. 5(b)-5(h) as green dashed lines have been obtained by considering a single pass though an ensemble with $\mathrm{OD}_{\text {tot }}=m \times \mathrm{OD}_{\mathrm{SP}}$. We also analyzed the exact experimental situation by including the optical cavity in the Hamiltonian of the system [solid red line in Figs. 5(b)-5(h); see Supplemental Material [18]). The predictions for these two theoretical formulations agree for our experimental settings, meaning that our system allows us to study waveguide-mediated infinite range interactions [5].

Our results promote nanofiber-coupled atomic ensembles as a unique platform to reveal the microscopic aspects of collective effects in a one-dimensional ensemble. We note that, while the atom-light coupling is only partially chiral in our system [27], the enhancement of forward emission typical of the timed Dicke state results in properties similar to a cascaded quantum system, in which emitters are only coupled to light which propagates in one direction [27]. Future research plans include extending this study beyond the single-excitation regime to explore the collective nonlinear response of coupled two-level systems $[7,28,29]$. From this point of view, a time-resolved analysis of nonclassical properties of the transmitted light, including, e.g., correlation among photons [30], squeezing [31], and multiphoton bound states [32] would certainly be of great interest. In addition, our nanofiber ring resonator with variable in- and out-coupling rate is an ideal candidate to investigate non-Markovian dynamics [23] as well as the physics of collective enhancement while continuously transitioning from the regime of waveguide quantum electrodynamics to cavity quantum electrodynamics.

We acknowledge financial support by the Alexander von Humboldt Foundation in the framework of an Alexander von Humboldt Professorship, endowed by the Federal Ministry of Education and Research, and by the Austrian Science Fund (NanoFiRe Grant Project No. P31115).

*riccardo.pennetta@hu-berlin.de

†Present address: Vienna Center for Quantum Science and Technology, Faculty of Physics, University of Vienna, 1090 Vienna, Austria.

*arno.rauschenbeutel@hu-berlin.de

[1] R. H. Dicke, Coherence in spontaneous radiation processes, Phys. Rev. 93, 99 (1954). 
[2] M. O. Scully and A. A. Svidzinsky, The super of superradiance, Science 325, 1510 (2009).

[3] M. O. Araújo, I. Krešić, R. Kaiser, and W. Guerin, Superradiance in a Large and Dilute Cloud of Cold Atoms in the Linear-Optics Regime, Phys. Rev. Lett. 117, 073002 (2016).

[4] S. J. Roof, K. J. Kemp, M. D. Havey, and I. M. Sokolov, Observation of Single-Photon Superradiance and the Cooperative Lamb Shift in an Extended Sample of Cold Atoms, Phys. Rev. Lett. 117, 073003 (2016).

[5] P. Solano, P. Barberis-Blostein, F. K. Fatemi, L. A. Orozco, and S. L. Rolston, Super-radiance reveals infinite-range dipole interactions through a nanofiber, Nat. Commun. 8, 1857 (2017).

[6] W. Guerin, M. O. Araújo, and R. Kaiser, Subradiance in a Large Cloud of Cold Atoms, Phys. Rev. Lett. 116, 083601 (2016).

[7] G. Ferioli, A. Glicenstein, L. Henriet, I. Ferrier-Barbut, and A. Browaeys, Storage and Release of Subradiant Excitations in a Dense Atomic Cloud, Phys. Rev. X 11, 021031 (2021).

[8] A. Goban, C.-L. Hung, J. D. Hood, S.-P. Yu, J. A. Muniz, O. Painter, and H. J. Kimble, Superradiance for Atoms Trapped along a Photonic Crystal Waveguide, Phys. Rev. Lett. 115, 063601 (2015).

[9] R. J. Bettles, T. Ilieva, H. Busch, P. Huillery, S. W. Ball, N. L. R. Spong, and C.S. Adams, Collective mode interferences in light-matter interactions, arXiv:1808.08415v4.

[10] S. Okaba, D. Yu, L. Vincetti, F. Benabid, and H. Katori, Superradiance from lattice-confined atoms inside hollow core fibre, Commun. Phys. 2, 136 (2019).

[11] A. Paris-Mandoki, C. Braun, J. Kumlin, C. Tresp, I. Mirgorodskiy, F. Christaller, H. P. Büchler, and S. Hofferberth, Free-Space Quantum Electrodynamics with a Single Rydberg Superatom, Phys. Rev. X 7, 041010 (2017).

[12] N. Stiesdal, H. Busche, J. Kumlin, K. Kleinbeck, H. P. Büchler, and S. Hofferberth, Observation of collective decay dynamics of a single Rydberg superatom, Phys. Rev. Research 2, 043339 (2020).

[13] R. Rohlsberger, K. Schlage, B. Sahoo, S. Couet, and R. Ruffer, Collective Lamb shift in single-photon superradiance, Science 328, 1248 (2010).

[14] T. Bienaimé, N. Piovella, and R. Kaiser, Controlled Dicke Subradiance from a Large Cloud of Two-Level Systems, Phys. Rev. Lett. 108, 123602 (2012).

[15] A. A. Svidzinsky, J.-T. Chang, and M. O. Scully, Dynamical Evolution of Correlated Spontaneous Emission of a Single Photon from a Uniformly Excited Cloud of $N$ Atoms, Phys. Rev. Lett. 100, 160504 (2008).

[16] J.-T. Shen and S. Fan, Theory of single-photon transport in a single-mode waveguide. I. Coupling to a cavity containing a two-level atom, Phys. Rev. A 79, 023837 (2009).

[17] W. Guerin, T. do Espirito Santo, P. Weiss, A. Cipris, J. Schachenmayer, R. Kaiser, and R. Bachelard, Collective Multimode Vacuum Rabi Splitting, Phys. Rev. Lett. 123, 243401 (2019).

[18] See Supplemental Material at http://link.aps.org/supplemental/ 10.1103/PhysRevLett.128.073601 for more details on the theoretical description, the time evolution of the atomic excitation probability, the experimental sequence, and the data used to estimate the pulse decay rate shown in Fig. 3.

[19] M. Blaha, A. Johnson, A. Rauschenbeutel, and J. Volz, Beyond the Tavis-Cummings model: Revisiting cavity QED with atomic ensembles, arXiv:2107.04583v1.

[20] D. Steck, Cesium $D$ line data, http://steck.us/alkalidata (2019).

[21] A. Johnson, M. Blaha, A. E. Ulanov, A. Rauschenbeutel, P. Schneeweiss, and J. Volz, Observation of Collective Superstrong Coupling of Cold Atoms to a 30-m Long Optical Resonator, Phys. Rev. Lett. 123, 243602 (2019).

[22] F. LeKien and K. Hakuta, Spontaneous radiative decay of translational levels of an atom near a dielectric surface, Phys. Rev. A 75, 013423 (2007).

[23] K. Sinha, P. Meystre, E. A. Goldschmidt, F. K. Fatemi, S. L. Rolston, and P. Solano, Non-Markovian Collective Emission from Macroscopically Separated Emitters, Phys. Rev. Lett. 124, 043603 (2020).

[24] P. Schneeweiss, S. Zeiger, T. Hoinkes, A. Rauschenbeutel, and J. Volz, Fiber ring resonator with a nanofiber section for chiral cavity quantum electrodynamics and multimode strong coupling, Opt. Lett. 42, 85 (2017).

[25] G. Sagué, E. Vetsch, W. Alt, D. Meschede, and A. Rauschenbeutel, Cold-Atom Physics Using Ultrathin Optical Fibers: Light-Induced Dipole Forces and Surface Interactions, Phys. Rev. Lett. 99, 163602 (2007).

[26] C. C. Kwong, T. Yang, M. S. Pramod, K. Pandey, D. Delande, R. Pierrat, and D. Wilkowski, Cooperative Emission of a Coherent Superflash of Light, Phys. Rev. Lett. 113, 223601 (2014).

[27] P. Lodahl, S. Mahmoodian, S. Stobbe, A. Rauschenbeutel, P. Schneeweiss, J. Volz, H. Pichler, and P. Zoller, Chiral quantum optics, Nature (London) 541, 473 (2017).

[28] A. Cipris, N. A. Moreira, T. S. do Espirito Santo, P. Weiss, C. J. Villas-Boas, R. Kaiser, W. Guerin, and R. Bachelard, Subradiance with Saturated Atoms: Population Enhancement of the Long-Lived States, Phys. Rev. Lett. 126, 103604 (2021).

[29] A. Angerer, K. Streltsov, T. Astner, S. Putz, H. Sumiya, S. Onoda, J. Isoya, W. J. Munro, K. Nemoto, J. Schmiedmayer, and J. Majer, Superradiant emission from colour centres in diamond, Nat. Phys. 14, 1168 (2018).

[30] A. S. Prasad, J. Hinney, S. Mahmoodian, K. Hammerer, S. Rind, P. Schneeweiss, A. S. Sørensen, J. Volz, and A. Rauschenbeutel, Correlating photons using the collective nonlinear response of atoms weakly coupled to an optical mode, Nat. Photonics 14, 719 (2020).

[31] J. Hinney, A. S. Prasad, S. Mahmoodian, K. Hammerer, A. Rauschenbeutel, P. Schneeweiss, J. Volz, and M. Schemmer, Unraveling Two-Photon Entanglement via the Squeezing Spectrum of Light Traveling through Nanofiber-Coupled Atoms, Phys. Rev. Lett. 127, 123602 (2021).

[32] S. Mahmoodian, G. Calajó, D. E. Chang, K. Hammerer, and A. S. Sørensen, Dynamics of Many-Body Photon Bound States in Chiral Waveguide QED, Phys. Rev. X 10, 031011 (2020). 